\title{
Disentangling the Dynamical Mechanisms for Cluster Galaxy Evolution
}

\author{
Xiaolei Zhang ${ }^{1,2}$ \\ ${ }^{1}$ Remote Sensing Division \\ US Naval Research Laboratory \\ Washington, DC 20375, USA \\ ${ }^{2}$ Current Affiliation: \\ Department of Physics and Astronomy \\ George Mason University \\ Fairfax, VA 22030, USA \\ xzhang5@gmu.edu
}

Received — 


\begin{abstract}
The determination of the dynamical causes of the morphological Butcher-Oemler (BO) effect, or the rapid transformation of a large population of late-type galaxies to earlier Hubble types in the rich cluster environment between intermediate redshifts and the local universe, has been an important unsolved problem which is central to our understanding of the general problems of galaxy formation and evolution. In this article, we survey the existing proposed mechanisms for cluster galaxy transformation, and discuss their relevance and limitations to the explanation of the morphological BO effect. A new infrared diagnostic approach is devised to disentangle the relative importance of several major physical mechanisms to account for the BO effect, and an example of the first application of this procedure to a single rich, intermediate redshift galaxy cluster is given to demonstrate the viability of this approach. The preliminary result of this analysis favors the interaction-enhanced secular evolution process as the major cause of the cluster-galaxy morphological transformation. This conclusion is also supported by a wide range of other published results which are assembled here to highlight their implications on a coherent physical origin for the morphological BO effect.
\end{abstract}




\section{Introduction}

Central to the study of the cosmic evolution of galaxies is the identification of the dominant dynamical mechanism responsible for the rapid transformation, between intermediate redshifts and the local universe, of cluster galaxies from a population characterized by a significant fraction of late-type spirals to one dominated by S0s and ellipticals: the so-called morphological Butcher-Oemler (BO) effect (Butcher \& Oemler 1978a,b; Dressler et al. 1997).

At the heart of the BO effect is the spiral to early-type transformation (Dressler et al. 1997; Ellis 1998). This transformation not only involves the extinguishment of the star-formation activity, but also the gradual increase of bulge-to-disk ratio (Franx 2004; Treu 2004 and the references therein). Furthermore, whichever physical mechanism(s) produced the BO effect, in addition to producing the observed number density evolution of cluster galaxies, must simultaneously be able to preserve the relatively tight color-magnitude relation observed throughout the redshift range $\mathrm{z}=0-1$ (van Dokkum \& Franx 2001). The relevant process for galaxy transformation must also preserve the outer disks and spiral structures observed for many of these galaxies during the quenching of star formation and the morphological transformation (Couch et al. 1998; Poggianti et al. 1999; Goto et al. 2003b).

Several candidate mechanisms have been proposed to account for the morphological BO effect. These include: major and minor mergers (Icke 1985; Kauffmann 1995), galaxy harassment through tidal shocks (Moore et al. 1996,1998,1999), galaxy infall onto the forming cluster and the resulting stripping of either the disk or halo gas (with the latter having the name of "starvation") due to the ram pressure of the intracluster medium (Gunn \& Gott 1972; Larson, Tinsley, \& Caldwell 1980), as well as a newly-discovered secular dynamical process mediated by the large-amplitude density wave patterns excited during the tidal interaction of galaxies in clusters (Zhang 1996,1998,1999; Zhang \& Buta 2007a,b).

The dominant mechanism responsible for producing the BO effect is still uncertain. Most of the previously-proposed mechanisms run into contradictions with one or another of the 
observed characteristics of cluster galaxies upon careful analyses. Due to the high-speed nature of galaxy-galaxy encounters within clusters which prevents the encountering galaxies from "sticking" to one another, major mergers are not believed to have a significant probability in clusters (Dressler et al. 1997), though an exception to this conclusion has been observed for one high-z cluster (van Dokkum et al. 1998). Moreover, there does not seem to be a large reservoir of dwarf spheroidal satellites in these clusters (Trentham 1997) to cause the simultaneous morphological transformation of the large number of $\mathrm{BO}$ galaxies by minor mergers. The tightness of the color-magnitude relation of cluster early-type galaxies (Franx 2004 and the references therein) and the well-preserved disks throughout the BO evolution process (Moran et al. 2007b and the references therein) also argue against a significant role played by mergers.

Ram pressure stripping of the interstellar medium for infalling cluster galaxies (Gunn \& Gott 1972) by itself appears not to be sufficient to account for the observed level of cluster galaxy transformation. Stripping of gas generally does not lead to a significant change in the bulge-to-disk (B/D) ratio, though Larson et al. (1980) had envisioned the reduction of disk size due to gas-stripping as one way of increasing the B/D. However, recent observations and analysis by Moran et al. (2007a) showed that cluster spiral galaxies have systematically higher central density than their field counterparts, an effect which is difficult to produce if the outer-disk stripping is the major cause of the cluster $\mathrm{BO}$ effect.

There is also the issue of timescale with any stripping/starvation mechanism which relies on the infall of galaxies from the field region into the centers of clusters: If an infalling galaxy starts its journey from the field at $5 \mathrm{Mpc}$ distance to the cluster center, and has an average velocity of $500 \mathrm{~km} / \mathrm{sec}^{1}$, it takes the galaxy $4 \times 10^{9}$ years to reach the central region. This is a significant

\footnotetext{
${ }^{1}$ Even though the cluster velocity dispersion is generally on the order of $1000 \mathrm{~km} / \mathrm{sec}$, the velocities of galaxies at the outskirts of the clusters, especially their radial components, are generally quite low, since these outer galaxies have not been virialized and thus have not gained the kind of high velocity dispersion that the cluster-center galaxies have.
} 
fraction of the Hubble time, and comparable to the cluster formation/evolution timescale. So the late-type galaxies observed in the central regions of clusters as found by Dressler et al. (1997) are likely to be primordial instead of new in-fallers from the field region. The existence of the morphology-density relation over several orders-of-magnitude variation of local surface density (Dressler 1980; Dressler et al. 1997; Goto et al. 2003c), from the densest region of cluster center all the way to the group environment in the field, also supports the segregation of the varying-density regions of a cluster during cluster-galaxy morphological transformation, and argues against significant mixing of field populations into the central regions of clusters. Fujita (2004) proposed the idea that galaxies could be "pre-processed" in groups and intermediate density regions, which then become mixed with the cluster in somewhat less time than the field-to-cluster-core infall time. However, adding a pre-processed piece of material onto the outskirts of the cluster does not have the same effect as mixing thoroughly the material of this newly-added piece into the entire cluster region including the central core. This could have allowed the preservation of the relative identities of the different cluster regions as the cluster grows and matures, as well as the maintenance of the observed morphology-density relation.

The galaxy harassment mechanism (Moore et al. 1996,1998,1999) has gained some popularity in recent years. When first proposed, harassment was meant as a process whereby the gravitational tidal shock from a large cluster galaxy tears away the outer disk of a small late-type neighboring galaxy, thus transforming the small galaxy into an early-type dwarf spheroidal galaxy. Numerical simulations by Moore et al. (1998) showed that the harassment process generates remnants which are predominantly prolate in shape, as opposed to the oblate shape that an S0 galaxy (which is the typical end-product of BO transformation) usually has. The follow-on exploration of the harassment mechanism by Gnedin $(1999,2003)$ showed that this mechanism is rather inadequate in transforming the morphology of large disk galaxies: even though a moderate puffing-up of the entire disk was observed, there is not a significant increase in the bulge-to-disk ratio which is a required element for the spiral-to-S0 transformation. The simulations conducted by Moore et al. (1999) showed that the low-surface-brightness galaxies in their tests were transformed to 
dwarf spheroical galaxies due to tidal shocks, whereas for high-surface-brightness galaxies "the scaleheight of the discs increases substantially and no spiral features remain". This is in marked contrast to the post-interacting galaxies observed by, e.g. Poggianti et al. (1999) and Moran et al. (2007b), which showed prominent spiral structures and well-preserved outer disks after whatever interaction processes which had resulted in the star-burst episodes in these galaxies.

Another proposed mechanism for producing the BO effect is the secular dynamical evolution process in galaxies which is further enhanced by the tidal interactions in the cluster environment

(Zhang 1996,1998,1999; Zhang \& Buta 2007a,b). In this scenario, tidal forces between neighboring galaxies and between a galaxy and the cluster potential serve to excite large-amplitude, open spiral or bar patterns. Most of the morphological transformation is realized through the post-interaction mass redistribution process due to the presense of tidally-enhanced density wave patterns. In this process the radial mass accretion throughout the galaxy disk and the vertical heating of stars occur simultaneously over the time span of a few Gyrs, due to the irreversible energy and angular momentum exchange between the density wave and the disk matter and the outward transport of these exchanged energy and angular momentum by the density wave to the outer disk, which lead to the gradual build-up of the bulge (Zhang 1999; Zhang \& Buta 2007a,b), in addition to the consumption of gas through density-wave induced star formation. This mechanism has been shown to be able to preserve the tight scaling relation of early-type galaxies during the secular mass accretion process (Zhang 2004).

\section{An Infrared Diagnostic Approach for the Star-Formation States of Cluster Galaxies}

In order to assess the relevance of the different proposed mechanisms to the explanation of the BO effect, we have developed an infrared (IR) diagnostic approach which has the potential to shed new light on the BO problem using the existing and soon-to-be-available data offered by the new generation of the space IR telescopes such as Spitzer and Herschel. 
Over the past three decades since the initial discovery of the BO effect, the observational studies of cluster galaxy evolution have been mostly through ground-based and space-based optical telescopes, owing to their much superior sensitivity, angular resolution, and area-covering efficiency. However, it is well known that optical observations suffer from dust extinction which can shield some of the most intense star-formation activities in clusters (Geach et al. 2006). On the other hand, the existing IR observations, even those done with the state-of-the-art space IR telescopes, are limited in their angular resolution compared to optical observations. For Spitzer Space Telescope observations of intermediate redshift galaxies, for example, essentially only the total flux across the different IR bands are known for each cluster galaxy, with no further spatial resolution within a single galaxy. Therefore, given this state of the current IR observations, the new method is devised to make use of only the bolometric lumonisty information (which is contributed most significantly by the IR bands for starburst galaxies) of cluster galaxies.

The method makes use of the fact that most of the proposed mechanisms for BO transformation will involve varying levels of dusty starbusrt, and furthermore these different mechanisms have different predicted characteristic IR luminosity levels, as well as different timescales fot sustaining these different IR luminosity levels. Given the known BO transformation rate (which can be determined from a statistical study of the galaxy morphologies in rich clusters at the intermediate redshifts, compared to a similar study for the local universe), we can infer the roles of different proposed mechanisms by observing the different frequecies of occurence of the varying IR-luminous galaxies in the different regions of intermediate clusters. This quantitative approach has major advantages in constraining the importance of the candidate dynamical mechanisms in transforming cluster galaxy morphologies compared to the simple-minded counting of the IR sources of a given luminosity range in an observing epoch, because the timescales of the operation of these dynamical mechanisms are vastly different, and a simple counting approach can result in serious misconceptions of the relative contributions of the different processes, as our example in the next section will show.

For a cluster region of total galaxy number $N$, if there are $n$ galaxies observed to be in a 
luminosity range characteristic of a type of starburst event, we denote the observed frequency for that type of event $f_{\text {event }}$ as

$$
f_{\text {event }}=\frac{n}{N}
$$

Assume the duration of the starburst event is $\tau_{\text {event }}$ (usually believed to be on the order of $10^{8} \mathrm{yr}$ for strong-interaction-type events such as merger and harassment, and much longer for gas-stripping type events and for post-interaction secular evolution), after a cluster evolutionary timescale of $T$ (on the order of a few Gyr, the exact number depending on the redshift $z$ of a cluster), we expect a fraction

$$
F=f_{\text {event }} \cdot \frac{T}{\tau_{\text {event }}}
$$

of the cluster galaxies to have gone through morphological change due to the particular type of star-forming event alone.

The total fraction $F$ for BO transformation between an intermediate redshift and the local universe, due to the yet-unsettled physical mechanism(s), is now fairly well determined for rich BO clusters (see, e.g. van Dokkum \& Frank 2001; Franx 2004), and its values is around 20\%, for example, between $z=0.4$ and $z=0$ (see for example Figure $4 \mathrm{a}$ of van Dokkum \& Franx 2001, where at $z=0.4$ the early type fraction is about 0.6 , and at $z=0$ the early type fraction is about 0.8 , which means a fraction of $20 \%$ of late-to-early transformation between $z=0.4$ and $z=0$ had happened). Since this $20 \%$ conversion fraction is for cluster as a whole, and in the later calculations we divide the cluster into three broad regions (post-virialization, active virialization, and outskirts), we estimate the morphological conversion fraction between $\mathrm{z}=0.4$ and $\mathrm{z}=0$ for the active virialization region to be $30 \%$ and for the cluster outskirts about $F=10 \%$. No estimates for the post-virialization region is given since the IR diagnostics are not available there, but it is expected that the morphological transformation process is going on at some level even in that region where the star formation has ceased.

We stress that not all of the mechanisms that can transform galaxies will include a dusty-starburst phase (for example, one proposed pathway for morphological transformation not 
involving a dusty starburst is dry mergers). Even for those mechanisms which can potentially invoke a dusty starburst, this phase will only be prominent when there is sufficient gas reservior in the galaxy at the observing epoch. This approach thus predicts the maximum possible abundance of dusty starbursts that can be attributed to each mechanism, to be compared with that of the observed abundance. So for the proposed approach to work effectively, we make the implicit assumption that the $\mathrm{BO}$ precursor galaxies are gas-rich galaxies, which seemed to be a valid one since that was how Butcher and Oemler first discoved them (i.e., by observing their blue colors due to enhanced star formation in a gas-rich environment). For the later stage of the evolution, after the gas is consumed, we can still have the interaction and the associated morphological transformation events but no star-formation signature. However, the arguments for the validity of the fractions estimated by this approach require only that the relevant galaxies are gas rich at the observing epoch z, and that the interaction strength of the relevant mechanisms stay roughly constnat throughout z. They do not require the gas fraction to remain the same throughout z. This is because we are relating the star-forming events at $\mathrm{z}$ with the overall morphological transformation rate $\mathrm{F}$ throughout $\mathrm{z}$, and the latter does not require a constant gas supply. So in this sense the star-formation events at the observation epoch $\mathrm{z}$ is only a diagnostic signature of the underlying morphological transformation process.

We therefore cannot rule out the possibility that there are other processes which potentially could be transforming galaxies in a way that does not generate an IR-bright phase, rather we are ruling out these mechanisms as the explanation for the observed incidence of dusty starbursts in those clusters where abundant starbursts are observed. In $\S 3$, we will show that the starbursts which are observed in one particular intermediate redshift cluster can essentially only be explained via the dynamical mechanism of interaction-enhanced secular evolution, which demonstrated the power of this approach.

On the other hand, using the same principle this kind of "frequency test" can in fact be used also for sources observed in other wave bands (such as optical), as long as there is a clearly-identifiable spectral/photometric feature which has a known timescale of operation. 
If the timescale for the individual interaction event $\tau_{\text {event }}$ is known, we can invert the above expression to calculate the appropriate value of $f_{\text {event }}$ for each type of interaction event in the appropriate luminosity range and for the relevant cluster region. These calculated results are tabulated in Table 1 for a hypothetical BO cluster redshift of $z \sim 0.4$. In this table we list calculated values of $f_{\text {event }}$ for each of the proposed physical mechanisms we consider, split into several radial cluster regions, and for transforming galaxies that fall into several different classes of IR luminosity. We will describe in more detail in the following subsections of how the frequencies in Table 1 are obtained.

Note that if the predictions/observations were made at a different $z$ (say $z=0.2$ instead of $z=0.4$ ), then the observed fraction of IR sources are expected to be lower due to the smaller amount of available gas (i.e. some of the galaxies have already completed the late-type to S0 converson between $z=0.2$ and $z=0.4)$. This effect is partly taken into account by the outward migration of the boundaries of the active virializing region with decreasing $\mathrm{z}$, partly by the corresponding change of $F(z)$ at the new observing epoch z. Of course part of the $F(z)$ dependence accounts for the reduced elapsed time for a smaller $\mathrm{z}$ as well. Yet the argument should hold no matter what observation epoch one uses, as long as one concentrates to the regions where virializating is going on. The gradually-enlarging central part of the cluster then falls into the N/A no-prediction zone.

Note also that in Table 1 we did not break the IR luminosities of the moderately-luminous galaxies at the usual LIRG (Luminous InfraRed Galaxies) level (which has the lowest luminosity value of $L_{I R}=10^{11} L_{\odot}$ ), but rather at $4 \times 10^{11} L_{\odot}$. This choice of division is due to our preliminary examination of the results of Geach et al. (2006)'s Spitzer $24 \mu m$ observations of cluster CL $0024+16$ at $z=0.39$. In this cluster the majority of the IR sources detected have colors intermediate between star-forming and passive galaxies, indicating that this is possibly a transitional-type population, and these transition-type galaxies have luminosities extending to about $4 \times 10^{11} L_{\odot}$.

Furthermore, in Table 1 we have divided each cluster into three approximate zones (Goto 
et al. 2003a): (1) The very central post-virialization region, where the star-formation activity has ceased by the epoch of observation. For this region we do not give predictions of IR bright sources, since we assume these are rare due to the exhaustion of the gas for galaxies in this region. However, some post-interaction morphological transformation could still be going on here after the extinguishment of star formation. (2) The active-virialization region. For this region the virialization-triggered star-formation activity is in full swing, as will be the star-forming activities due a number of proposed mechanisms. For clusters at different redshift ranges, this intermediate zone has been found to propagate from the inner cluster toward outer cluster region as redshift decreases. (3) The cluster outskirts. In this region the star formation activity is elevated compared to the field, but not as vigorous as in the active virialization region.

The predictions for the frequencies of IR bright sources are thus given only for the latter two regions of clusters, since for the first region all of the proposed mechanisms can be made compatible potentially with the observation of a lack of star-formation activity there. The proposed observed fractions for regions (2) and (3) are further divided into sub-categories for each proposed dynamical mechanism (assuming it operates alone, or is the dominating one in a particular region).

\subsection{Galaxy Merger}

In the popular cold dark matter $(\mathrm{CDM})$ paradigm, galaxy mergers are the preferred means of morphological evolution of galaxies in clusters (see, e.g., Kauffmann 1995). Even though the likelihood of mergers in the dense regions of clusters has been questioned because of the encounter-speed arguments, the issue of to what extent mergers play a role in cluster galaxy evolution, in both the dense regions as well as in the outskirts, have never been firmly settled, especially due to the paucity of high angular resolution mid- and far-IR observations of distant clusters before the launch of Spitzer Space Telescope.

For major mergers between large, gas-rich disk galaxies, we expect to see ultraluminous 
infrared galaxies (ULIRGs) with peak IR luminosities $L_{I R}$ in the range of $10^{12}-10^{13} L_{\odot}$ based on the local UILRGs observations, though lower luminosity-ranged mergers had been observed as well presumably between smaller galaxies or less gas-rich systems (Sanders \& Mirabel 1996, Table 3). The assumptions we made to arrive at the numbers used in Table 1 for mergers are that the galaxies involved in these mergers are large, as well as gas-rich disks, a condition clearly satisfied by the BO-effect progenitor galaxies (Dressler et at. 1997). For the virializing region of a dense cluster, if we expect major mergers to be responsible for producing the observed level of BO effect, or the transformation of $\mathrm{F}=30 \%$ of the galaxy population from spirals to S0s and other early types in the past $5 \mathrm{Gyr}$, and if we assume an interaction timescale of $5 \times 10^{7}-1.5 \times 10^{8}$ yr for each of the two higher luminosity ranges listed in Table 1 (Sanders \& Mirabel 1996, who further cited the merger simulations of Mihos \& Hernquist 1994; also Murphy et al. 1996), equation 2 gives an observed merger fraction of $f_{\text {merger }}=0.3-0.9 \%$ for each of the higher IR luminosity ranges. We have also assumed the BIRGs (bright IR galaxies, with $L_{I R}$ in the range of $\left.6 \times 10^{10} L_{\odot}-4 \times 10^{11} L_{\odot}\right)$ phase to last about twice as long as the average VBIRGs (very bright IR galaxies, with $L_{I R}$ in the range of $\left.4 \times 10^{11} L_{\odot}-10^{12} L_{\odot}\right)$ and ULIRGs phases, i.e., it has a timescale of $2 \times 10^{8} \mathrm{yr}$, tracing precursors and aftermaths of merger events, which leads to the estimate of $f_{\text {merger }}=1.2 \%$ in the BIRG phase.

We note that different individual merger events have different interaction kinematic configurations, which will also result in the difference in how long the merging process will last. This uncertainty in the timescales of merging events should not hamper our ability to determine the possible roles of merger in a given cluster, since the predictions of the merger scenario, both in terms of observed fractions and in terms of the luminosity of its most luminous members, are orders of magnitude different from other scenarios, and thus can tolerate this level of uncertainty. In the example we present in the next section, we will see that the predominence of moderately-luminous IR sources in that cluster from many different angles precludes the possibility that merger had played a significant role in transforming the galaxy morphologies in that cluster.

For the outskirts of the cluster we assumed have a morphological transformation rate of 
$F=0.1$. This leads to an average $f_{\text {merger }}=0.2 \%$ expected for the outskirts if merger is the major cause of the BO transformation, slightly higher than for the field of $f_{\text {merger }}=0.1 \%$ which is the observed value (Sanders \& Mirabel 1996).

\subsection{Galaxy Harassment}

Since galaxy harassment is a mechanism proposed based on numerical simulations (Moore et al. 1996,1998), rather than on observations of physical processes, the precise timescales of this mechanism are not prescribed. We expect, from the general dynamics of high-speed swing-by between galaxies, that the timescale should be on the order of $10^{8} \mathrm{yr}$ for each of the BIRG and VBIRG phases, similar to the less-violent phases of a major merger. Based on this assumption, as well as the proposed multiple nature of the harassing encounters, which we take to be 5 for an average galaxy to go through the late-to-early-type transition through the harassment process, we arrive at an overall time scale $t_{\text {event }}$ of $10^{9}$ years ( 5 times of $10^{8}$ years in each of the two luminosity ranges), which is reasonable considering that this is essentially the timescale of the cluster-core crossing for cluster galaxies. The high-density cluster core region is believed to be where the harassment mechanism is most effective (Moore 2004). These timescales gave the estimates of the observing frequencies quoted in Table 1.

The harassment process is expected to be much less effective away from the high-density cluster core regions, since it depends on the strong tidal interaction between close-neighbor galaxies. We therefore set the expected observing frequency for IR-bright galaxies due to harassment in the outskirts of clusters to be nearly zero. Any observed IR bright galaxies in those regions must be due to physical processes other than galaxy harassment. We would not expect to see ULIRGs either in the core or in the outskirts due to the action of galaxy harassment process alone. 


\subsection{Ram Pressure Stripping}

Like the harassment mechanism, the ram pressure stripping mechanism is also expected to operate mostly in the dense region of a cluster, since that is where the intracluster medium (ICM) is mostly concentrated. If ram pressure is the dominant mechanism for the BO transformation, we would expect BIRGs to be preferentially located in the central virializing region of clusters, since this is a gentle process which is not expected to produce ultra-bright IR sources. Furthermore, the timescale of operation of this mechanism is on the order of a cluster-core crossing time, or about $10^{9}$ yr (Bosseli \& Gavazzi 2006), though shorter timescales (down to $10^{8}$ years) have been proposed as well (Quilis et al. 2000; Fujita \& Nagashima 1999). This leads to the frequencies of observations derived for the active virialization region and the outskirts of a cluster due to the operation of ram-pressure stripping alone, as shown in Table 1. We would not expect to see ULIRGs in any regions of the cluster due to the ram pressure stripping process alone.

\subsection{Post-Interaction Secular Evolution}

The post-interaction secular evolution process refers to the enhanced star-formation and radial mass accretion process mediated by the large-amplitude, open density wave patterns such as spirals, bars, as well as skewed three-dimensional mass distributions (Zhang 1999; Zhang \& Buta 2007a,b). Secular evolution can operate in both the core as well as the outskirts of a cluster (or even in the group environment or in isolation), the only difference between these environments is the different evolution rates caused by the different strengths of the density waves, which in turn are caused by the different amount of tidal perturbation in these environments.

For the tidally-enhanced secular evolution process we expect a large population of BIRGs with luminosities up to the lower range of VBIRGs. The IR manifestations of the secular evolution process depend not only on the strength of tidal perturbation, but also on the availability of gas supplies for star-formation. In the post-virialization region, even though the mass accretion process may still be going on, the star formation activity can be significantly quenched due to the 
paucity of gas in this region of the galaxy disk.

For the active virializing region, since the member galaxies are effectively always feeling the gentle tidal nudges of the near and as well as far neighboring galaxies (a condition characterizing the virialization itself), we therefore set the highest timescale of secular evolution in the BIRGs phase to be $5 \times 10^{9}$ yr, i.e., exactly equal to the amount of elapsed time between $\mathrm{z}=0.4$ and $\mathrm{z}=0$. This of course assumes the continuous availability of gas during this time period. To take into account of the more rapid consumption of gas (i.e., a shorter star-formation timescale than the morphological-transformation timescale), we set the lower limit to be half of the maximum. Note that these timescale estimates for secular evolution assumes spiral-induced star formation and mass accretion which are a disk-specific processes. There could exist more violent primordial, clumpy mass accretion at higher $\mathrm{z}$ due to dynamical friction in proto-galaxies, as might be what have terminated the star formation in large and early-virialized clusters such as MS0451, which we will discuss later.

For the outskirts we have assumed the star formation timescale to be about $1.5 \times 10^{9}$ yr, typical of post-starburst evolution timescale (Poggianti et al. 1999), since the interaction frequency at the outskirts is expected to be low, and interaction is not continuous as in the active virialization region.

With these assumptions we derive the expected fractions of the BIRG sources as given in Table 1. The fraction of the VBIRGs are calculated assuming an interaction timescale of $10^{8} \mathrm{yr}$, describing the precursor starburst events leading to some of the subsequent secular evolution. We would not expect to see ULIRGs due to the secular evolution process alone.

Finally we want to comment here that in this entire derivation for the frequency table (Table 1), we have implicitly assumed: (1) the constancy of the rates of the interaction processes across the redshift range under concern, (2) the availability of the gas supply to feed the star-formation activity at the epoch of the observation $\mathrm{z}$, and (3) a single process dominates the BO transformation. All of these assumptions may need to be modified, at least for some clusters 


\begin{tabular}{|c|c|c|c|c|}
\hline & $f_{\text {merger }}$ & $f_{\text {harassment }}$ & $f_{\text {gas stripping }}$ & $f_{\text {secular evolution }}$ \\
\hline $\begin{array}{c}\text { post-virialization } \\
\text { region }\end{array}$ & $\mathrm{N} / \mathrm{A}$ & $\mathrm{N} / \mathrm{A}$ & $\mathrm{N} / \mathrm{A}$ & $\mathrm{N} / \mathrm{A}$ \\
\hline $\begin{array}{c}\text { active } \\
\text { virializing } \\
\text { region }\end{array}$ & $\begin{array}{c}\sim 1.2 \% \text { BIRGs } \\
(12) \\
\sim 0.3-0.9 \% \text { VBIRGs } \\
(3-9) \\
\sim 0.3-0.9 \% \text { ULIRGs } \\
(3-9)\end{array}$ & $\begin{array}{c}\sim 3 \% \text { BIRGs } \\
\quad(30) \\
\sim 3 \% \text { VBIRGs } \\
\quad(30) \\
\sim 0 \% \text { ULIRGs } \\
(0)\end{array}$ & $\begin{array}{c}\sim 0.6 \%-6 \% \text { BIRGs } \\
(6-60) \\
\sim 0 \% \text { VBIRGs } \\
(0) \\
\sim 0 \% \text { ULIRGs } \\
(0)\end{array}$ & $\begin{array}{c}\sim 15-30 \% \text { BIRGs } \\
(150-300) \\
\sim 0.6 \% \text { VBIRGs } \\
(6) \\
\sim 0 \% \text { ULIRGs } \\
(0)\end{array}$ \\
\hline outskirts & $\begin{array}{c}\sim 0.4 \% \text { BIRGs } \\
(4) \\
\sim 0.1-0.3 \% \text { VBIRGs } \\
(1-3) \\
\sim 0.1-0.3 \% \text { ULIRGs } \\
(1-3)\end{array}$ & $\begin{array}{c}\sim 0 \% \text { BIRGs } \\
(0) \\
\sim 0 \% \text { VBIRGs } \\
(0) \\
\sim 0 \% \text { ULIRGs } \\
(0)\end{array}$ & $\begin{array}{c}\sim 0 \% \text { BIRGs } \\
(0) \\
\sim 0 \% \text { VBIRGs } \\
(0) \\
\sim 0 \% \text { ULIRGs } \\
(0)\end{array}$ & $\begin{array}{c}\sim 3 \% \text { BIRGs } \\
(30) \\
\sim 0.2 \% \text { VBIRGs } \\
(2) \\
\sim 0 \% \text { ULIRGs } \\
(0)\end{array}$ \\
\hline
\end{tabular}

Table 1: The expected fractions and numbers of galaxies to be observed in each luminosity range for $\mathrm{z} \sim 0.4$ rich clusters in two different cluster environments, for different proposed physical processes acting alone, generated using equation (2). The expected number of detected galaxies for the different scenarios are given in parentheses, assuming 10 clusters, each having an average of 200 galaxies in the two outer regions (and therefore approximately 100 galaxies in each of the two regions described above). Here ULIRGs (UltraLuminous InfraRed Galaxies) have IR luminosities in the range of $L_{I R}=10^{12}-10^{13} L_{\odot}$, (Sanders \& Mirabel 1996), VBIRGs (Very Bright InfraRed Galaxies) have $L_{I R}=4 \times 10^{11}-10^{12} L_{\odot}$, and BIRGs (Bright InfraRed Galaxies) have $L_{I R}=6 \times 10^{10}-4 \times 10^{11} L_{\odot}$. 
and during some periods of the evolution. So corroborating data from other wavelengths for the clusters under study will help to arrive at a satisfactory picture of the major drivers for the morphological evolution in a given cluster, or in a population of clusters studied in a statistical sense. Apart from these implicit assumptions the only uncertainty of the Table quantities comes from the timescale value $\tau_{\text {event }}$ for each proposed physical process (since $F$ and $T$ in equation 2 are well-determined quantities and are common for all processes in a given cluster).

\section{An Example of the Application of the Proposed Approach}

The recent 24 micron Spitzer observations of two distant clusters (Geach et al. 2006) have revealed elevated levels of star formation throughout the cluster up-to and slightly-beyond the cluster turn-around radius, both compared with that determined using optical observations and compared to values typical of the field environment at the same redshift. It also revealed very different frequencies of IR-luminous sources in these two clusters: For MS 0451-03 at $\mathrm{z}=0.55$, very few $24 \mu \mathrm{m}$ sources were detected; whereas for CL $0024+16$ at $\mathrm{z}=0.39$, a large excess of the $24 \mu \mathrm{m}$ sources were detected ( 150 IR galaxies over a 25'x25' area, or 9x9 $\left.\mathrm{Mpc}^{2}\right)$.

The difference in the rate of IR luminous sources may reflect a correlation of the IR sources with the virialization state of the cluster, i.e., even though MS 0451-03 is at slightly higher z than CL $0024+16$, it has larger mass and also appears to be at a more advanced stage of virialization than CL 0024+16 (Geach et al. 2006; Moran et al. 2007b), and therefore the most intense star-formation episodes may have already been over by the observation epoch (as evidenced by the presense of many passive spiral galaxies in this cluster, Moran et al. 2007b). In this case the entire cluster belongs to region 1 (post-virialization region) of the Table, which can be consistent with essentially all of the proposed mechanisms (i.e., we will not be able to discriminate among the proposed mechanisms for this particular cluster). To sum up, the lack of IR sources in MS 0451 may indicate the operations of early-epoch physical processes (such as clumpy mass accretion due to dynamical friction or spherical mass accretion) prior to the observational epoch which had 
effectively suppressed star formation before any other processes (such as secular evolution) would have an opportunity to generate an IR-bright starburst at the observational epoch, though the mass accretion process due to stellar density wave structures in the passive spirals in this cluster could still continue to modify galaxy morphology without generating the corresponding starburst activity. The star-formation condition of MS 0451 is not expected to be typical for an average BO cluster at intermediate redshifts, though, since BO clusters are usually seleted to contain a large population of gas-rich star-forming galaxies. We will focus our attention of the application of the proposed approach thus only on CL $0024+16$.

It is well known that in disk galaxies star formation happens mostly around the spiral arms or bars, i.e., around the density wave crest. This is because the disk is usually stable to the gravitational collapse of matter, and a density wave shock (which is a collisionless shock induced by the nonlocal gravitational potential wave which is phase-shifted with respect to the density distribution) is needed to trigger a violent episode of star formation (Zhang 1996 and the references therein). The reason that interactions usually enhance the amplitude of the spiral density wave is because the spontaneous growth rate of the density wave is usually very low (Zhang 1998), so many field spirals can have very low amplitude density waves, and remain in the disk phase even until the recent time, whereas in the cluster environment, the tidal interactions remove angular momentum from the disk galaxies, and also excite large seeds of non-axisymmetric perturbations which are further amplified by the disk's intrinsic modal resonance mechanism.

Most of the $24 \mu \mathrm{m}$ galaxies in CL $0024+16$ observed by Geach et al. (2006) show enhanced luminosity in the range of BIRGs, or $6 \times 10^{10} L_{\odot}$ to $4 \times 10^{11} L_{\odot}$, compared to their counterparts in the field $\left(\sim 2-3 \times 10^{10} L_{\odot}\right.$ for normal disk galaxies, see Sanders \& Mirabel 1996). The majority of the $24 \mu \mathrm{m}$ sources detected by Geach et al. (2006) displays an optical/NIR colors that are intermediate between star-burst galaxies and passive galaxies, which indicates that this is likely a population of galaxies in the post-interaction stage, and are going through the slow and prolonged evolution under the influence of the interaction-enhanced density-waves. We now apply the frequency approach developed in the last section to further confirm the nature of these $24 \mu \mathrm{m}$ 
sources.

Geach et al. (2006) found that there are about 150 IR luminous sources in an area of 25'x25', the majority of them are in the IR luminosity range of $6 \times 10^{10}-2 \times 10^{11} L_{\odot}$, which, compared with the total galaxy number of about 500 determined by Moran et al. (2005) for the same region, and taking into account of completeness estimates $(\sim 65 \%$ completeness for galaxies in the range of $17.75<I<21.1$ and $\sim 40 \%$ for galaxies in the range of $17.75<I<22.5$ ), leads to an estimation of a fraction of about $15 \%$ of IR-bright sources among the entire optical sample ${ }^{2}$. Note that Geach et al. (2006) did not carry out the luminosity statistucs of their $24 \mu \mathrm{m}$ sources according to the different radial ranges in the cluster, but rather estimated the luminosity statistics for the cluster as a whole. Therefore we do not have more detailed information at the present time to do a more detailed analysis in terms of the radial distributions of the frequency of the different kinds of IR bright sources.

The derived fraction of IR-bright sources is in the correct range as predicted by Table 1 for secular evolution to account for the majority of the BO transformation between the intermediate redshift and the present for this cluster: the fraction predicted in Table 1, when averaged over the entire cluster region, would lead to a predicted BIRG fraction of around 15\%. From Geach

\footnotetext{
${ }^{2}$ In arriving at these fractional estimates we have considered the fact that we might need to extend the lower bound of the IR flux range from $6 \times 10^{10} L_{\odot}$, which is the $5 \sigma$ detection limit of the Geach et al. observations, to closer to $3 \times 10^{10} L_{\odot}$ which is the typical flux level of the local dusty starburst galaxy, such as NGC 253. However, we note that not all dusty starburst at the lower IR luminoisty range will lead to the conversion of a late-type to an early-type galaxy in $5 \mathrm{Gyr}$, therefore the more numerous IR sources at the lower luminosity range probably should not enter into the current statistics. This consideration is also supported by the recent result of Elbaz et al. (2007) who found higher redshift starburst galaxies to be much more IR luminous on average than the local starburst galaxies of similar morphology.
} 
et al. (2006)'s result, it is also seen that the brighter IR sources (those in the VBIRG range of $\left.4 \times 10^{11}-6 \times 10^{11} L_{\odot}\right)$ consist of about 4 sources, or $0.4 \%$, which is also consistent with the secular evolution scenario. Most significantly, there were no sources detected above the flux level of $6 \times 10^{11} L_{\odot}$, or no ULIRGs or strong interactions present in this cluster at this observing epoch. Improved statistics from a much larger sample would help to quantitatively assess the roles of other proposed mechanisms across the entire population of BO clusters.

Dressler et al. (1999) showed that the post-starburst galaxy fraction in their sample of 10 intermediate redshift clusters observed using the HST in the MORPHS project is about 20\%, similar to the fraction of IR-bright sources in CL 0024+16. Poggianti et al. (1999) confirmed that the total $\mathrm{a}+\mathrm{k} / \mathrm{k}+\mathrm{a}$ type galaxy(the type of emission-line galaxies signifying a post-starburst population) fraction is about $20 \%$. They had had considerable trouble identifying the progenitor population of the post-starburst galaxies, however, presumably because these last only a brief period and therefore are less numerous.

In the MORPHS sample analyzed by Poggianti et al. (1999) and Dressler et al. (1999), 10\% of cluster galaxies were clasified as e(a) (which is a dusty-starburst spectral class), and about $40 \%$ of these (or $4 \%$ of the total) showed morphological signs of tidal interaction or were observed to be directly involved in a merger/close interaction. This is much larger that the maximum allowed fraction of mergers we had estimated in Table 1 (i.e., $0.9 \%$ ), which was calculated based on the observed $\mathrm{BO}$ transformation rate between $\mathrm{z}=0.4$ and the local universe. We therefore felt that what Poggianti et al. and Dressler et al. observed could be not all mergers but rather interactions that were confused with mergers due to limited spatial resolution. This conclusion is also supported by the fact that in both CL 0024 and MS 0451, the Spitzer 24 um MIR observations by Geach et al. (2006) had revealed essentially no ULIRGs population at all.

There exists the question of whether the few highest luminosity sources (currently estimated to be in the mid range of $10^{11} L_{\odot}$ in the Geach et al. observations) in CL 0024 could have been undergoing mergers, because there is uncertainty in the conversion of $24 \mu \mathrm{m}$, or the rest frame $15 \mu m$ flux to the IR luminosity. We argue that this possibility could not be true for this cluster, 
based on the entire distribution of the IR sources in this cluster. In Table 1 the predicted rates for mergers are not only for the ULIRGs, but also for the BIRGs and VBIRGs. For a given dynamical scenario the IR source statistics in all three ranges of the IR luminosity need to be accounted for, not just the highest luminosity range. The over-abundance of moderately IR-bright sources in this cluster is not consistent with a scenario in which merger is the dominant process for BO transformation in this cluster, because if so a roughly 0.3-0.9\% ULIRG fraction would by itself be able to account for the $\mathrm{F}=30 \% \mathrm{BO}$ conversion, which leave the rest of the $15 \%$ moderately luminous IR sources unaccounted for. Or if these moderately bright IR source also lead to transformation by some other dynamical mechanisms such as secular evolution in addition to the role played by merger, together they would be over-producing the observed $30 \%$ BO conversion ratio between $\mathrm{z}=0.4$ and present.

Furthermore, the observed IR sources could also not have been consistent with their being the product of either harassment or ram pressure stripping, since these should have produced fewer IR sources based on the values given in Table 1. Plus, harassment should have erased the spiral structures whereas most of these post-interacting galaxies were found to be spiral disk galaxies (Poggianti et al. 1999; Moran et al. 2007b). Ram pressure stripping would also have difficulty producing the brighter portion of these IR sources in CL 0024. Thesefore, tidally-enhanced secular evolution process becomes the only possible remaining candidate to account for the origin of the population of IR sources in CL 0024.

Other recent ground-based optical observations on CL 0024 using other instruments appear to corroborate our conclusions about the role of interaction-enhanced secular evolution in accounting for these excess IR sources. Moran et al. (2007a) found from the analysis of resolved optical spectroscopy of CL 0024 galaxies and a control sample of field galaxies at similar redshifts that the cluster Tully-Fisher relation exhibits higher scatter than its field counterpart. They also found that the central mass densities of the spiral galaxy population they examined were higher within the cluster virial radius than outside, with a sharp break exactly at the cluster virial radius. The cluster environment thus appears to be responsible for the creation of the increase in scatter of 
the Tully-Fisher relation, as well as for the increase in central density of cluster spirals within the cluster virial radius.

Moran et al. (2007a)'s tentative explanation of these observations is that galaxy harassment process has eliminated the less-dense spirals in the cluster central regions. This explanation however could not account for the lack of high-central-density spirals in the cluster outskirts. So they proposed that these cluster-outskirts high-density spirals might have been eliminated by mergers. However, an obvious problem with this proposed explanation is why mergers in cluster outskirts would selectively eliminate only the high-density spirals but not the low-density spirals. Furthermore, $24 \mu \mathrm{m}$ observations of this cluster have not revealed a significant merging population in this cluster.

The observations of Moran et al. (2007a) were also difficult to explain through any kind of ram-pressure striping mechanisms, as these usually lead to unusual star formation gradient, whereas the enhanced scatter observed in Tully-Fisher relation was present in both the V and the $K_{s}$ band, and thus is not due to the influence of star-formation or dust obscuring, but rather has to be related to the kinematic and structural changes of the cluster galaxies, as Moran et al. (2007) had concluded.

The observed trend would be most consistent with a mild level of tidal perturbation for cluster galaxies (and these perturbations are obviously more pronounced for galaxies within virializing region of the cluster, because the condition of virialization essentially guarantees a continuous interacting state of the member galaxies in the cluster virial radius), as well as the post-interaction secular evolution of the galaxy morphology and kinematics.

The secular evolution scenario could naturally produce the observed difference in the central mass density of disk galaxies inside and outside the cluster virial radius, as well as the increase in structural and kinematic scatter of these disks (Moran et al. 2007a). Note however that the increase of scatter is only observed for the Tully-Fisher relation of the late-type cluster galaxies within the cluster virial radius. For S0s, for example, the fundamental scaling relation in fact 
becomes tighter within the virial radius (Illingworth et al. 2000). This phenomenon is easily explained in the secular evolution scenario as the settling onto an "attractor" of the dynamical evolution (see $\S 5)$.

Furthermore, the fact that the blue members of a cluster show more scatter when placed on a scaling relation plot, whereas the red ones show better correlation, shows that the interactions in the cluster environment tends to disturb the perfect Tully-Fisher relation for late-type cluster galaxies compared to the field. However, as the secular evolution proceeds, the cluster environment in fact produces the tightest scaling relations at the late stages of evolution, much tighter than the counterpart of these scaling relations for the field early-type galaxies. The tidal agitation in the cluster environment is a way to disturb one dynamical equilibrium (that of late type disks) to facilitate the settling onto a new dynamical equilibrium (that of early-type morphology, which is of higher gravitational entropy).

Once again, we emphasize that the conclusions we have reached based on the analysis of CL $0024+16$ serves only to illustrate the application of the approach we proposed, and the conclusion on the dominating mechanism to account for the $\mathrm{BO}$ effect for the majority of the intermediate redshift clusters can only be reached once we have analyzed enough samples of rich clusters by the same approach. At the time of this writing, CL 0024 and MS 0451 are the only intermediate-redshift clusters that have been observed in this fully-sampled wide-field fashion among the Spitzer-approved observations. A few more will be observed by Spitzer in Cycle 4 . Numerous other observations do exist, however, for the central, virialized cores of clusters, and can be used to further carry out the existing program. As we have seen in this preliminary study, even the information obtained in the core region already gave plenty of hints at the underlying dynamical mechanism responsible for cluster galaxy transformation, though the addition of the cluster ourskirts and group observations will help to complete the picture. 


\section{Other Corroborating Evidence in Support of a Secular Evolution Origin for the Morphological Butcher-Oemler Effect}

\subsection{Results from Sloan Digital Sky Survey}

Goto (2005) analyzed the velocity dispersion of 355 galaxy clusters (with 14548 member galaxies) from the Slone Digital Sky Servey (SDSS), and found that bright cluster galaxies have significantly smaller velocity dispersion than faint galaxies, consistent with a picture of dynamical friction (galaxy-galaxy interaction) operating during the process of cluster virialization which reduces the velocity dispersion of massive cluster galaxies at the expense of the increase of velocity dispersion of the less massive cluster galaxies. He also found that star-forming late-type galaxies in his sample have a larger velocity dispersion than the passive late-types (i.e. those having spiral morphology, but do not show any ongoing star-formation activity, see Goto et al. 2003b), which is

once again consistent with dynamical friction reducing the velocity dispersion of the more evolved (passive) population of galaxies.

The result of Goto (2005) is consistent with our conclusion reached from CL 0024+16 that tidally-enhanced secular evolution appears to be the driver for transforming the cluster galaxy morphology, since the galaxy-galaxy interaction and dynamical friction is known to excite large-amplitude density waves which leads to subsequence radial accretion of mass and the evolution of galaxy morphological types (Zhang \& Buta 2006). The Geach et al. (2006) and Goto et al. (2003b), Goto (2005) results are also mutually consistent in the sense that the $24 \mu \mathrm{m}$ sources which show intermediate colors between star-burst and passive populations are likely to be the population in transition from star-burst to passive galaxies, and these passive galaxies, through further secular evolution, will become the earlier Hubble-type cluster galaxies observed in nearby clusters.

The role of dynamical friction during the cluster relaxation process is also reflected in the often sharp transition in galaxy properties across the cluster virial radius, or at a critical local surface density (Moran et al. 2005,2007; Tanaka et al. 2004; Couch, Colless, \& Propris 2004. 
Figure 5 of Illingworth et al. 2000 shows another example of the change of the tightness of scaling relation for the S0 population across the cluster virial radii). These characteristics, as we commented before, can be naturally explained by the enhanced secular evolution due to the tidally-induced interaction processes.

Goto (2005)'s result is on the other hand inconsistent with ram pressure having played a significant role in causing cluster galaxy evolution. The effectiveness of the ram pressure is proportional to $\sigma v^{2}$ (where $\sigma$ is the gas density and $v$ is the velocity dispersion), which should be more effective for galaxies with larger velocity dispersion, i.e., it should have predicted that the larger-velocity-dispersion population is the more evolved one (i.e. passive), contrary to what is observed.

\subsection{Results from the MORPHS Collaboration}

The MORPHS team conducted imaging and spectroscopic studies of 10 distant clusters in the redshift range of 0.37 to 0.56. Poggianti et al. (1999) found that among the MORPHS cluster galaxies a significant over-abundance of the so-called "post starburst" galaxies with the characteristic $\mathrm{k}+\mathrm{a} / \mathrm{a}+\mathrm{k}$ spectral features, which exhibit spatial and kinematic distributions intermediate between the passive and active populations. The number density of these poststarburst population is significantly higher in their sample clusters than in the field, so there the star-burst activities prior to the $\mathrm{k}+\mathrm{a} / \mathrm{a}+\mathrm{k}$ phases were obviously triggered by the cluster environment, so are the subsequent termination of the star-forming events.

One interesting thing noticed by Poggianti et al. is the mostly spiral-like disky morphology of these post-starburst galaxies from the HST images, showing that whatever interaction processes which triggered the starburst had not changed the galaxy morphology immediately. This fact is contrary to both the merger and the harassment predictions, which require immediate change to galaxy morphologies after the interaction. The evidence for the milder tidal perturnation, on the other hand, is entirely consistent with an interaction-enhanced secular evolution scenario. 
Poggianti et al. also concluded that either two timescales or two different physical processes has caused the two observed transformation processes (the halting of star formation and the spiral-to-S0 transformation). This result is supported by the later investigation of Goto et al. (2004) using the SDSS data, who found that the color evolution of the BO galaxies in their sample is much faster than the morphologial evolution. In the secular evolution scenario, these two different timescales acquire a most natural explanation: the interaction events which serve to excite the large-amplitude spiral density waves and bars may only last on the order of 0.1-1 Gyr, but the subsequent secular mass accretion process could continue for much longer, i.e., could be a significant fraction of a Hubble time. Furthermore, the same process could operate on the passive phase of the galaxies involved as well, even when most of the star-formation activities have extinguished.

\section{Further Comments on the Different Proposed Mechanisms for Cluster Galaxy Evolution}

\subsection{Hierarchical Clustering and Mergers}

The Hierarchical clustering/CDM paradigm has been our standard paradigm for structure formation over the past few decades. However, growing evidence has pointed to the inadequacies of using this paradigm to explain the observations especially on the properties of individual galaxies.

The well known down-sizing trend of galaxy formation, not only in terms of star formation history but also in terms of mass assembly history (Cowie et al. 1996; Kodama et al. 2004, Cimatti, Daddi \& Renzini 2006 and the references therein) is the most obvious contradictory evidence to the bottom-up assembly scenario which is the foundation of the CDM paradigm. While the former trend (down-sizing in star-formation) can perhaps be accounted for by proposed mechanisms such as AGN or star-formation feedback (see, e.g., de Lucia et al. 2006; Croton et

al. 2006 and the references therein), the latter trend of mass assembly down-sizing (Cimatti et al. 2006) is in direct conflict with the mass assembly history predicted by the CDM paradigm. 
The observed early formation age of cluster early type galaxies (Franx 2004 and the references therein), even after taking into account some late formations due to the BO effect, is also contrary to the predictions of the CDM paradigm. CDM is known to significantly under-predict the extremely-red-objects (ERO) observed in the early universe (Daddi, Cimatti, \& Renzini 2000 and the references therein). It also predicted significant difference in the properties of the field and cluster early-type populations (Kauffmann 1996), which is not observed. Other evidence for the difficulty of using this paradigm to explain the observed properties of galaxies can be found in Zhang (2003).

CDM predicted the late assembly of early type galaxies, including cluster galaxies, which superficially is consistent with the morphological BO effect and with the increase in number density of early type galaxies observed for both the cluster and field (Treu 2004 and the references therein). However, unequal-mass mergers completely destroy the color-magnitude relation (Bower, Kodama \& Terlevich 1998), whereas equal-mass merging among massive galaxies reduces the early-type number density, contrary to the observed early-type number density evolution. Furthermore, collisionless mergers simply can't reproduced the high phase space density observed for the central regions of early-type galaxies (Ostriker 1980). The rotationally-supported S0 disk, which is the end product of the majority of BO transformation in clusters, is also problematic for a merger-based morphological transformation process. These observational constraints, coupled with the high velocity dispersion of cluster galaxies, means that mergers, including dry mergers, could not have played an important role in the BO transformation, a conclusion consistent with our finding in this work based on the analysis of the properties and frequencies of the IR sources for CL 0024. The recent analysis by Elbaz et al. (2007) on the GOODS fields data also directly confirmed that a great portion of the star-formation events at $z \sim 1$ is confined within single disk galaxies, rather than due to the merging of different galaxies. The star-formation rates observed in the GOODS fields also have drastically different dependence on the densities of galaxies than that predicted by the CDM paradigm (Elbaz et al. 2007, Fig. 8). The observed density dependence of star-formation rate, however, is consistent with the expectation from the interaction-enhanced 
secular evolution scenario.

\subsection{Infall, Stripping, Mixing, and More on the CDM Paradigm}

Poggianti et al. (1999) found no evidence among the MORPHS cluster galaxies of a difference in the radial distributions of passive and emission-line spiral galaxies, though the recent result of Moran et al. (2007b) is somewhat in conflict with it. A difference of distribution in the distribution of these two populations is to be expected if significant infall had occurred. Cooper et al. (2006,2007) described the results from the DEEP2 survey, and found that the steepening of the morphology-density relation works in the group environment just as effectively as in the cluster environment. The results from SDSS and 2dFGRS also support this conclusion. Therefore, cluster-specific mechanism, such as ram-pressume stripping, will not be a justified cause for the group galaxy evolution. Dressler et al. (1997) concluded that the morphology-density relation indicated that more local processes are at work. Couch, Matthew and De Propris (2004) reached similar conclusion from the $2 \mathrm{dF}$ survey results.

The new results of Elbaz et al. (2007) showed that at the high redshift the mechanism which relates galaxies to their environment is not simply a quencher but also a trigger of star formation: the star-formation/density relation at $\mathrm{z}=1$ in fact reverses the morphology-density relation trend. These authors concluded that their results are most consistent with a scenario where star formation is accelerated by the dense environment of galaxies, resulting in the faster exhaustion of their gas reservoir. This new result of the star-formation and galaxy environment dependence is inconsistent with the ram-pressure stripping picture.

As we have commented earlier, the existence of the morphology-density relation (Dressler

1980; Dressler et al. 1997) means that there could not have been a significant amount of persistent mixing of late type galaxies from the field environment into the cluster environment during the cluster evolution process. Similar minimal-mixing conclusions have also been reached by Abraham et al. (1996) and Morris et al. (1998). The hierarchical assembly scenario, on the other hand, 
advocates the gradual assembly of higher density clusters from lower density sub-clusters, which is slightly different from the continuous infall scenario for individual galaxies.

Through numerical simulations, Dressler (2004) and collaborators found that under the CDM paradigm there is actually significant mixing on cluster dynamical timescales, at least on the group level. This predicted mixing by the CDM, however, is in conflict with the observational evidences quoted above, including the existence of the morphology-density relation. This in fact from another side highlights the inadequacy of one of the central assumptions of the CDM paradigm (see, e.g., Peebles 1993): that of cluster formation as a purely gravitational process. This assumption is also what went into all the cluster CDM simulations which resulted in the observed mixing of the galaxy populations in the different regions of a cluster, contrary to the observational evidence of well-segregated evolution manifested in the morphology-density relation.

There are in fact very well established observational evidence that the cluster formation process is not a purely gravitational process. Evrard (2004) reviewed many existing theoretical and observational tests of cosmology using galaxy clusters as probes, and highlighted the deficiency of the cluster physics according to the CDM paradigm as "a cluster energetics problem". Under the assumption that the principle mechanism of ICM heating is through gravitationally induced shocks, there is about a $70 \%$ excess heat that could not be accounted for between what is required for virial equilibrium and what is observed. Furthermore, galaxy velocity dispersion in clusters shows a comparable level of excess "heat" compared to the dark matter velocity dispersion normalized to the WMAP and large scale structure distributions. Therefore, the cluster environment is in general not in a gravitationally unstable collapsing configuration as predicted by the CDM theories. Depending on gravitational collapse alone most of the clusters as we know would not have formed. Many of the observed clusters form through their inherent hydrodynamical large-scale systematic velocities and the resultant high-speed sub-cluster clump mergers (such as what had happened for CL 0024, as well as for many other well-known clusters in the intermediate redshifts as studied by the MORPHS collaboration and by Couch et al.), but the cause of these mergers are not gravitational collapse, but rather hydrodynamical collision due 
to their inherent relative velocities. A portion of the observed clusters such as MS 0451 do indeed appear to form out of gravitational collapse, however. These clusters are likely to be situated in a high density region in the primordial mass fluctuation spectrum, and thus are much better virialized as a whole because they satisfy better the gravitational instability condition. This latter class will appear to be more X-ray luminous for a given amount of baryonic mass. They also tend to evolve faster in terms of member galaxy morphologies as the example of MS 0451 had shown.

The excess systematic velocities of many of the proto-cluster clumps were thought to have originated from the so-called "primordial turbulence" at the time of CMB decoupling, an idea which can be traced back to the early proposals of von Weisacker (1951) and Gamow (1952), and which was subsequently substantiated by Ozernoy (1974a,b, 1978). This idea has recently been shown to be relevant also to the explanation of the angular spectrum of the the observed cosmic microwave background radiation (Bershadskii \& Screenivasan 2002,2003). Peebles (1974, Figure 1) had plotted the covariance function for the angular distribution of galaxies, and found that a power law fluctuation index $n$ between -1 and 1 can fit the observational angular distribution of galaxies. A power law index of $n=0.33$ can originate from the Komogorov type of turbulence velocity spectrum, and the dissipation in turbulence can steepen the residual velocity spectrum above $1 / 3$, to close to the WMAP observed value of $\mathrm{n} \approx 1$. If the primordial turbulence underlies the formation of the large scale structure and the clusters, then the paradoxial excess heat of the ICM and excess velocity dispersion of the cluster galaxies (Evrard 2004 and the references therein) would all acquire a very natural explanation, which the current CDM paradigm and the gravitational collapse picture failed to provide an explanation. In the primordial turbulence picture mergers due to inherent systematic velocities between proto-cluster clumps will play a much more important role, and infall and mixing which are associated with the CDM gravitational collapse will not be an important physical process during the cluster formation and evolution process.

Even though the CDM paradigm has been successful in explaining many features of the large-scale structure observations (though by no means this is the only possible way to explain it, 
as our above references had shown), the observations on galaxy-scaled phenomena is the area that the CDM paradigm has run into the most serious problems, both in terms of the mass profiles of galaxies along the Hubble sequence, for which it predicted the progressively-later Hubble types to have a more cuspy mass density, exactly opposite to what was observed, which shows that it is the early types that possess the cuspy surface density; as well as in terms of the mass assembly history, which is currently found to be anti-hierarchical (Cimatti et al. 2006). Secuclar evolution cannot be simply inserted into the CDM paradigm to help solve its many problems on the galaxy level, as many had hoped, because as an initial condition secular evolution assumes a physical low surface brightness (LSB) galaxy surface density profile, which is flat across the disk, not the cuspy type predicted by the CDM as a result of cold dark matter quickly sinking into the center of a galaxy.

\subsection{Galaxy Harassment}

Countrary to the later usage by many investigators, the early proposers of harassment intended it mainly as a mechanism for the transformation of dwarf disk galaxies to dwarf spheroidal galaxies (Moore et al. 1996). The harassment mechanism has been shown to be ineffective during the simulations for transforming the morphologies of large disks, especially in increasing the bulge-to-disk ratio (Gnedin 1999; Moore et al. 1999). As is well known, a large fraction of the disk galaxies undergoing BO transformation are normal spiral galaxies which have large disks (Dressler et al. 1997).

Furthermore, harassment is expected to be effective only in the dense regions of clusters (i.e. virialized cores) since it depends on multiple high-speed encounters, whereas the morphologydensity relation is shown to hold all the way from the dense core, to cluster outskirts, and to the group environment (Dressler 1980; Dressler et al. 1997; Cooper et al. 2007).

Moreover, harassment mechanism is expected to operate by tearing apart the outer disks of a galaxy, or at least destorying the spiral structure on the disk, and yet most of the cluster post-burst population have been found to have well-preserved disks which contain spiral structures 
(Poggianti et al. 1999; Couch et al. 1998; Goto et al. 2003b; Moran et al. 2007b).

We also want to emphasize that despite of some superficial similarities of the harassment mechanism and tidally-enhanced secular evolution, the harassment simulations in fact produced very different remnants as a result of interaction compared to interaction-enhanced secular evolution. For example, Moore et al. (1999) states that for LSB galaxies "the bound stellar remnants closely resemble the dwarf spheroidal (dEs) that populate nearby clusters", whereas for high surface brightness (HSB) galaxies "although very few stars are stripped, the scaleheight of the discs increases substantially and no spiral features remain". It is clear that those dEs are not related to cluster S0s which are the end product of BO type evolution. But even for the HSB galaxies, we know from the work of Poggianti et al. (1999) and Geach et al. (2007b) that the spiral structure and thin disks survive the star-formation events induced by interactions. So these remnants cannot be the same as those from the harassment simulations, which had their spiral structure completely destoryed by the strong tidal shocks. Furthermore, since secular evolution depends precisely on the presence of density wave features (i.e., spiral arms, bars, etc.) in the disks of galaxies to induce radial mass accretion, the operation of tidally-enhanced secular evolution requires a completely different galaxy-disk physical condition (cold disk with prominent density wave structure) than what the harassment mechanism can supply (the hot and puffed up disk devoid of density waves), despite the superficial similarities of these two proposed mechanisms.

\subsection{Secular Evolution and the Origin of Galaxy Scaling Relations}

In the secular evolution scenario, as a galaxy evolves along the Hubble sequence from the late to the early types, the increase in its mean surface brightness is accompanied by a corresponding decrease in the dynamical mass to light ratio (Broeils, Haynes, \& Baumgardt 1993; van der Hulst et al. 1993; Zwaan et al. 1995; Bell \& de Jong 2001; Zavala et al. 2003; and the references therein), these two effects compensating each other to maintain the tight scaling relations such as the Tully-Fisher and fundamental plane relations (Zhang 2004). 
The empirical color-magnitude relation (CM, see, e.g. Bower, Lucey \& Ellis 1992 and the references therein) has long puzzled astronomers as to its origin, especially in the face of obvious morphological transformation of galaxies as reflected in the BO effect and cluster early-type galaxy number density evolution (Franx 2004). The existence of this relation in the face of morphological transformation, however, could also naturally follow from the secular evolution process. The aging and reddening of the stellar population together with the gradual turning on of the star-forming mass reservoir (which increases the total luminosity of a galaxy) keep the galaxies on the CM relation as they redden and get more luminous. The conventional wisdom had always been that the constant slope of CM relations across redshift $\mathrm{z}$ means that this is not an age-mass relation, but rather a metalicity-luminosity relation (Ellis et al. 1997; Kodama et al. 1998), and the fact that most intermediate and nearby cluster early-type galaxies fall on tight color-magnitude relation means that their stars formed at high redshift (Franx 2004). This, however, implicitly assumed that the total luminosity $L$ of a galaxy does not increase with time. When the density-wave induced secular evolution process is considered, which results in a continued ignition of more and more baryonic star-forming material, as well as a continuous radial inflow of baryons to increase the effective mass of the early type galaxies, the total luminosity $L$ of a galaxy can increase with time. Although with the aging and reddening of the stellar population, the $M / L$ ratio of the early type galaxy increases (i.e. the average stellar population dims with age), the total $L$ can nonetheless increase because the increase of $\mathrm{M}$ due to secular evolution is much more substantial.

Observationally, it was found that many elliptical galaxies contain substantial outer disks (Franx 2004 and the references therein). The $\mathrm{L}^{*}$ disky ellipticals are found to form a continuous sequence with the S0s. Thus the continuous secular evolution through disk-related mass accretion process can conceivably lead to the increase of the elliptical galaxies' mass throughout the Hubble sequence. For the cluster early-type population, it was found by Fasano et al. (2000) that for clusters which have a large elliptical population, their S0 population is correspondingly reduced, and vice versa. This gives indirect support to the idea that the secular evolution process can in fact carry an early type galaxy across the S0 Hubble type into disky ellipticals. 
So far, high-z surveys have not uncovered many red cluster-galaxy progenitors, presumably this means they are yet to be transformed into such. The thought-to-be high-z progenitors of the nearby early type galaxies, the Lyman break galaxies (Steidel et al. 1996), were found to be much less massive than the average early type galaxies in the nearby universe (Papovich, Dickinson, \& Steidel 2001; Shapley et al. 2001). It is likely that the mass of these high-z early type galaxies have grown significantly since these earlier epochs through the secular mass accretion process. This explanation is one way to account for the "progenitor bias" as well as the increase in number density of early type galaxies with decreasing redshift (Treu 2004 and the references therein).

The explanation of the nature of the color-magnitude relation as effectively an age-mass relation resolves the dilemma faced by the conventional explanation, that while on the one hand the observed morphological and spectroscopic BO effect for cluster galaxies means that not all cluster galaxy stars are old, on the other hand the CM relation in the nearby universe is so tight which would have implied a uniform high formation redshift of the major population of stars in these early type cluster galaxies by the conventional wisdom. This explanation also naturally accounts for the fact that for every cluster observed (even those in the nearby universe), there are always some blue outliers which were off the main track of the cluster CM relation. This shows that galaxies only settle onto the CM relation as they age, since when starting off they are generally not already on the CM relation. The CM relation is thus another "attractor" of the secular evolution process.

This new explanation of the meaning of color-magnitude relation is also consistent with the recently-established down-sizing trend (see, e.g. Cimatti, Daddi, \& Renzini 2006; Kodama et al. 2004 and the references therein) for structure formation and mass assembly, which requires that the oldest object to be the most massive ones: this, coupled with the empirical CM relation, means that the red objects are indeed older, in addition to being metal rich. The most recent observations have by now given direct confirmation that the colors of the early-type galaxies in the local universe are indeed correlated with the mean age of the stellar population (Barr et al. 2007). 


\section{Conclusions}

In this paper we have reviewed the exising dynamical mechanisms to account for the origin of the cluster galaxy morphological transformation, and pointed out serious contradictions to observed properties of cluster galaxies when many of these mechanisms are examined in detail. We proposed further a new approach of using panoramic space infrared observations to quantitatively assess the roles of the different proposed mechanisms in transforming the cluster galaxy morphology and in producing the Butcher-Oemler effect. The preliminary application of this approach, coupled with a wealth of published results analyzed under the new light, points to interaction-enhanced secular evolution process as the most likely contributing mechanism for producing the morphological Butcher-Oemler effect. This conclusion is supported, most importantly, by the following observational evidence: that the interaction-induced morphological transformation process in clusters appears to be a disk-related process with well-preserved density wave patterns present on the galaxy disk through most of the evolution process; that the transformation appears to go through a univeral sequence of starburst, poststarburst, passive spiral, S0, and disky elliptical stages; that the quenching of star-formation appears to happen on a shorter timescale than the corresponding morphological transformation timescale; that the mid-infrared signature of the interacting events shows the predominence of a large population of moderately infrared-bright galaxies, which have optical and near-infrared colors as well as number densities consistent with them being the population in the transitional stage between starburst and passive galaxies. All of the above evidence are consistent with the interaction-enhanced secular evolution scenario for galaxy morphological transformation in clusters.

\section{Acknowledgments}

The author is indebted to J. Fischer, W. Couch, A. Dressler, C. Dudley, E. Dwek, E. Egami,

G. Helou, J. Huang, A. Oemler, D. Sanders, S. Satyapal, J. Schombert, and J. Virtelek for helpful comments and input to this project during the preparation of a Spitzer proposal to further explore 
the application of the IR diagnostic procedure described in this work. Thanks to S. Moran for making available the optical-spectroscopic-redshift dataset on the member galaxies in CL $0024+16$. An anonymous refereee provided a very thorough review of the manuscirpt, and many of his helpful suggestions are incorporated into the current text. J. Fischer proof-read a significant fraction of the manuscript and has made valuable comments towards its improvement. Thanks to E. van Kampen and collaborators for incorporating some of the elements of the current work in a new Herschel proposal to test the cluster galaxy evolution scenarios. This research was supported in part by funding from the Office of Naval Research.

\section{References}

Abraham, R. et al. 1996, ApJ, 471, 694

Barr, J.M., Bedregal, A.G., Aragon-Salamanca, A., Merrifield, M.R., \& Bamford, S.P. 2007, arXiv/astroph-0705.0623

Bershadskii, A., \& Sreenivasan, K.R. 2002, Phys. Lett. A, 299, 149

Bershadskii, A., \& Sreenivasan, K.R. 2003, Phys. Lett. A, 319, 21

Boselli, A., \& Gavazzi, G. 2006, PASP, 118, 517

Bower, R.G., Kodama, T., \& Terlevich, A. 1998, MNRAS, 299, 1208

Bower, R.G., Lucey, J.R., \& Ellis, R.S. 1992, MNRAS, 254, 601

Butcher, H., \& Oemler, A. Jr. 1978a, ApJ, 219, 18

- 1978b, ApJ, 226, 559

Cimatti, A., Daddi, E., \& Renzini, A. 2006, A\&A, 453, L29

Contursi, A. 1998, in Untangling Coma Berenices: A New Vision of an Old Cluster, eds. Mazure, A., Casoli, F., Durret, F., Gerbal, D., p. 203 
Cooper et al. 2006, MNRAS, 370, 198

Cooper et al. 2007, MNRAS, 376, 1445

Couch, W.J., Matthew, M.C., \& De Propris, R. 2004, in Clusters of Galaxies: Probe of

Cosmological Structure and Galaxy Evolution, eds. J.S. Mulchaey, A. Dressler, \& A. Oemler (Cambridge: CUP)

Couch, W.J., Ellis, R.S., Sharples, R., \& Smail, I. 1994, ApJ, 430, 121

Couch, W.J. et al. 1998, ApJ, 497, 188

Couch, W.J., \& Sharples, R.M., MNRAS, 229, 423

Cowie, L.L., Songaila, A., Hu, E.M., \& Cohen, J.G. 1996, AJ 112, 839

Daddi, E., Cimatti, A., \& Renzini, A. 2000, A\&A, 362, 45

Dale, D.A., \& Helou, G. 2002, ApJ, 576, 159

Dressler, A. 1980, ApJ, 236, 351

Dressler, A. et al. 1997, ApJ, 490, 577

Dressler, A., Smail, I., Poggianti, B.M., Butcher, H., Couch, W.J., Ellis, R.S., \& Oemler, A., Jr. 1999, ApJS, 122, 51

Duc, P.-A., et al. 2002, A\&A, 382, 60

Elbaz, D., Daddi, E., Le Borgne, D., Dickinson, M., Alexander, D.M., Chary, R.-R., Starck, J.-L., Brandt, W.N., Kitzbichler, M., MacDonald, E., Nonino, M., Popesso, P., Stern, D., \& Vanzella, E. $2007, A \& A, 468,33$

Ellis, R.S., Smail, I., Dressler, A., Couch, W.J., Oemler, A., Jr., Butcher, H., \& Sharples, R.M. 1997, ApJ, 483, 582

Evrard, A.E. 2004, in n Clusters of Galaxies: Probes of Cosmological Structure and Galaxy Evolution, eds. J.S. Mulchaey, A. Dressler, and A. Oemler, p.1 (Cambridge: CUP) 
Faber, S.M., \& Jackson, R.E. 1976, ApJ, 204, 668

Fadda, D. et al. 2000, A\&A, 361, 827

Fasano, G., Poggianti, B.M., Couch, W.J., Bettoni, D., Kjaerggard, P., \& Moles, M. 2000, ApJ, 542,673

Franx, M. 2004, in Clusters of Galaxies: Probes of Cosmological Structure and Galaxy Evolution, eds. J.S. Mulchaey, A. Dressler, and A. Oemler, p.196 (Cambridge: CUP)

Fujita, Y. 2004, PASJ, 56, 29

Fujita, Y. \& Nagashima,, M. 1999, ApJ, 516, 619

Gamow, G., 1952, Phys. Rev. D, 86, 251

Geach, J.E. et al. 2006, ApJ, 649, 661

Gnedin, O.Y. 1999, in Galaxy Dynamics, eds. D. Merritt, J.A. Sellwood, \& M. Valluri (San Francisco: ASP), 495

Gnedin, O.Y. 2003a, ApJ, 582, 141; 2003b, ApJ, 589, 752

Goto, T. 2005, MNRAS, 359, 1415

Goto, T., Okamura, S., Yagi, M., Sheth, R.K., Bahcall, N.A., Zabel, S.A., Crouch, M.S., Sekiguchi, M., Annis, J., Bernardi, M., Chong, S.-S., Gomez, P.L., Hansen, S., Kim, R.S.J., Knudson, A., McKay, T.A., \& Miller, C.J. 2003a, PASJ, 55, 739

Goto, T., Okamura, S., Maki, S., Bernardi, M., Brinkmann, J., Gomez, P.L., Harvanek, M., Kleinman, S.J., Krzesinski, J., Long, D., Loveday, J., Miller, C.J., Neilsen, E.H., Newman, P.R., Nitta, A., Sheth, R.K., Snedden, S.A., Yamauchi, C. 2003b, PASJ, 55, 757

Goto, T., Yamauchi, C., Fujita, Y., Okamura, S., Sekiguchi, M., Smail, I., Bernardi, M., Gomez, P.L. 2003c, MNRAS, 346, 601

Goto, T., Yagi, M., Tanaka, M., Okamura, S. 2004, MNRAS, 348, 515 
Gunn, J.E., \& Gott, J.R. 1972, ApJ, 176,1

Hansen, L. et al. 2000a, A\&A, 356, 83; 2000b, A\&A, 362, 133

Icke, V. 1985, A\&A, 144, 115

Illingwoth, G., Kelson, D., van Dokkum, P., \& Franx, M. 2000, Ap\&SS, 269, 485

Kauffmann, G. 1995, MNRAS, 274, 153

Kauffmann, G. 1996, MNRAS, 281, 487

Kodama, T. et al. 2004, MNRAS, 350, 1005

Kodama, T., Arimoto, N., Barger, A.J., \& Aragon-Salamanca, A. 1998, A\&A, 334, 99

Larson, R.B., Tinsley, B.M., \& Caldwell, C.N. 1980, ApJ, 237, 692.

Lemonon, L., Pierre, M., Cesarsky, C.J., Elbaz, D. ,Pello, R.; Soucail, G.; Vigroux, L, 1998, A\&A, $334, \mathrm{~L} 21$

Mann, R.G. et al. 1997, MNRAS, 289, 482

Martini, P. et al. 2006, ApJ, 644, 116

Mihos. J.c., \& Hernquist, L. 1994, ApJL, 431, 9

Moore, B. 2004, in Clusters of Galaxies: Probes of Cosmological Structure and Galaxy Evolution, eds. J.S. Mulchaey, A. Dressler, and A. Oemler, p.295 (Cambridge: CUP)

Moore, B., Katz, N., Lake, G., Dressler, A., O emler, A., Jr. 1996, Nature, 379, 613

Moore, B., Lake, G., \& Katz, N. 1998, ApJ, 495, 139

Moore, B., Lake, G., Quinn, T., \& Stadel, J. 1999, MNRAS, 304, 465

Moran, S.M., Ellis, R.S., Treu, T., Smail, I., Dressler, A., Coil, A.L., \& Smith, G.P., 2005, ApJ, 634,977 
Moran, S.M., Miller, N., Treu, T., Ellis, R.S., \& Smith, G.P. 2007a, ApJ, 659, 1138

Moran, S.M., Ellis, R.S., Treu, T., Smith, G.P., Rich, R.M., \& Smail, I. 2007b, ApJ, in press (astroph/0707.4173)

Morris, S.L., Hutchings, J.B., Carlberg, R.G., Yee, H.K.C., Ellingson, E., Balogh, M.L., Abraham, R.G., Smecker-Hane, T.A. 1998, ApJ, 507, 84

Murphy, T.W.,Jr., Armus, L., Matthews, K., Soifer, B.T., Mazzarella, J.M., Shupe, D.L., Staruss, M.A., \& Neugebauer, G. 1996, AJ, 111, 1025

Ostriker, J. 1980, Comments on Astrophysics, 8, 177

Ozernoy, L.M., 1974a, in The Formation and Dynamics of Galaxies, ed. J.R. Shakeshaft (IAU), 85

Ozernoy, L.M., 1974b, in Confrontation of Cosmological Theories with Observational Data, ed. M.S. Longair, 227

Ozernoy, L.M., 1978, in Confrontation of Cosmological Theories with Observational Data, ed. M.S. Longair (IAU), 227

Papovich, C., Dickinson, M., \& Ferguson, H.C. 2001, ApJ, 559, 620

Peebles, P.J.E. 1974, ApJL, 189, 51

Peebles, P.J.E. 1993, Principles of Physical Cosmology (Princeton: Princeton Univ. Press)

Poggianti, B.M., Smail, I., Dressler, A., Couch, W.J., Barger, A.J., Butcher, H., Ellis, R., \& Oemler, A., Jr. 1999, ApJ, 518, 576

Quilis, V., Moore, B., \& Bower, R. 2000, Science, 288, 1617

Rakos, K. \& Schombert, J. 2005, AJ, 130, 1002

Rowan-Robinson, M. et al. 1997, MNRAS, 289, 490

Sanders, D.B., \& Mirabel, F.H. 1996, ARAA, 34, 749 
Shapley, A.E., Steidel, C.C., Adelberger, K.L., Dickinson, M., Giavalisco, M., \& Pettini, M. 2001, ApJ, 562, 95

Smail, I., Dressler, A., Couch, W.J., Ellis, R.S., Oemler, A., Jr., Butcher, H., \& Sharples, R.M. 1997, ApJS, 110, 213

Steidel, C.C., Giavalisco, M., Dickinson, M., \& Adelberger, K.L., AJ, 112, 353

Tanaka, M., Goto, T., Okamurka, S., Shimasaku, K., \& Brinkman, J. 2004, in Outskirts of Galaxy Clusters: Intense Life in the Suburbs, Proc. IAUC 195, ed. A. Diaferio, p.444

Tran, K.-V. H., Franx, M., Illingworth, G., Kelson, D.D., i \& van Dokkum, P. 2003, ApJ, 599, 865

Trentham, N.A. 1997, PhDT, Univ. of Hawaii

Treu, T. 2004, in Clusters of Galaxies: Probes of Cosmological Structure and Galaxy Evolution, eds. J.S. Mulchaey, A. Dressler, and A. Oemler, p.177

Tully, R.B., \& Fisher, J.R. 1977, A\&A, 54, 661

van Dokkum, P. et al. 1998, ApJL, 504, 17

van Dokkum, P. \& Franx, M. 2001, ApJ, 553, 90

von Weizsacker, C.F. 1951, ApJ, 114, 165

Zhang, X. 1996, ApJ, 457, 125

Zhang, X. 1998, ApJ, 499, 93

Zhang, X. 1999, ApJ, 518, 613

Zhang, X. 2003, JKAS, 36, 223

Zhang, X. 2004, Ap\&SS, 319, 317

Zhang, X., \& R.J. Buta, 2007a, in Proc. IAUS 235, Galaxy Evolution across the Hubble Time, eds. F. Combes and J. Palous, p. 184 
Zhang, X., \& R.J. Buta, 2007b, AJ, 133, 2584 